\title{
A review about Smart Objects, Sensors, and Actuators
}

\author{
Cristian González García, Daniel Meana-Llorián, B. Cristina Pelayo G-Bustelo, and Juan Manuel Cueva Lovelle
}

University of Oviedo, Department of Computer Science, Oviedo, Spain

\begin{abstract}
Smart Objects and the Internet of Things are two ideas which describe the future, walk together, and complement each other. Thus, the interconnection among objects can make them more intelligent or expand their intelligence to unsuspected limits. This could be achieved with a new network that interconnects each object around the world. However, to achieve this goal, the objects need a network that supports heterogeneous and ubiquitous objects, a network where exists more traffic among objects than among humans, but supporting for both types. For these reasons, both concepts are very close. Cities, houses, cars, machines, or any another object that can sense, respond, work, or make easier the lives of their owner. This is a part of the future, an immediate future. Notwithstanding, first of all, there are to resolve a series of problems. The most important problem is the heterogeneity of objects. This article is going to show a theoretical frame and the related work about Smart Object. The article will explain what are Smart Objects, doing emphasis in their difference with NotSmart Objects. After, we will present one of the different object classification system, in our opinion, the most complete.
\end{abstract}

Keywords - Smart Objects, Internet of Things, Sensors, Actuators.

\section{What IS AN OBJect?}

$\mathrm{T}$ HROUGHOUT the difference literature about the universe of the Internet of Things, we could see the word 'object' in general. Why? The reason is simple. The word 'object' is used to refer to any device or thing, which can be intelligent or not. It means that when people talk about the interconnection among objects they talk about the interconnection among Smart Objects, among Not-Smart Objects, or between both.

Nevertheless, there are a lot of problems in the literature and the people's understanding when the word 'object' is said, furthermore, some people use the word 'thing' instead of 'object', or some authors use both words interchangeably. The reason is that both definitions are very ambiguous due to the use of these word in the articles or our daily life. This is why, in this first section, we are going to introduce the exact meaning in order to delete this ambiguity.

\section{Object according to WordReference [1]}

1. Anything that can be seen or touched and is for the most part stable or lasting in form, and is usually not alive.

2. A thing, person, or matter to which thought or action is directed; the cause of such thought or action.

Thing according to WordReference [1]

1. An object, usually not a person or animal.
Object according to Oxford [2]

1. A material thing that can be seen and touched.

Thing according to Oxford [2]

1. An object that one need not, cannot, or does not wish to give a specific name.

Object according to Cambridge [3]

1. A thing that can be seen or felt.

Thing according to Cambridge [3]

1. An object; something that is not living.

As we could see, the definition depends on the site where you consult and, even so, in these cases, the definitions can be very ambiguous. The definition that is better adapted for the typical use of the word 'object' in the universe of the Internet of Things is the first definition of 'object', the definition obtained from WordReference. Based on this, one possible definition to the word 'object' in the universe of the Internet of Things could be:

Any electronic device that can be connected to the Internet and collect data, like a sensor, or perform an action in an object, normally called actuator.

In the following sections, we will detail the differences between Smart Objects and Not-Smart Objects, explaining what are their and using examples of each one.

\section{Not-Smart OBJects}

In the previous section, we explained what the objects are and what elements compose this group. These elements are the objects without intelligence and objects with intelligence which are also known as Smart Objects. Due to the existence of these elements, it is essential to know how to distinguish the different type of objects and know the way in which these objects can interact with us. In this section we will address the objects of the second group, the objects without intelligence or Not-Smart Objects, and in later sections we will deepen in the Smart Objects. The Not-Smart Objects can be formed by sensors and actuators.

Sensors are electronic devices composed of sensitive cells [1] that are able to measure physical parameters like the light fluctuation using a photoresistor, the temperature using a thermistor, to detect flames, sounds, movements, or any other fluctuation in the environment [1], [4]. Thus, sensors are specific physical elements that allow us to 
measure a concrete physical parameter or detect something of the sensor's immediate environment.

However, actuators can be mechanic actuators which allow actions over themselves or over other devices, and actions which a specific object allow to perform. Thus, we can divide actuators in two different groups: mechanic devices and actions. Examples of mechanic actuators could be motors, servomotors or hydraulic bombs, and examples of actions could be to send a message, control LEDs, turn on lights or control the movement of a robot or any other available robot's actions.

According to the previous definitions, we could find devices that combine both types of Not-Smart Objects, they not only would have actuators and sensors but also would have both. An example of these are smartphones or any other Smart Object that are composed by sensors and actuators. Another similar example could be a microcontroller like an Arduino. The Arduino microcontroller is capable of manage almost any type of electronic device. Thus, an Arduino allows creating a system composed only of actuators, only of sensors, or both. Therefore, the Smart Objects are formed by Not-Smart Objects.

Fig. 1 shows a concept map that explains the composition of the objects. This Fig. is useful to understand better the difference between Not-Smart Objects and Smart Objects. As we can see in Fig. 1, NotSmart Objects can be sensors or actuators, and actuators are divided into mechanic actuators and actions. Moreover, in order to improve the understandability, Fig. 1 shows several examples of each group.

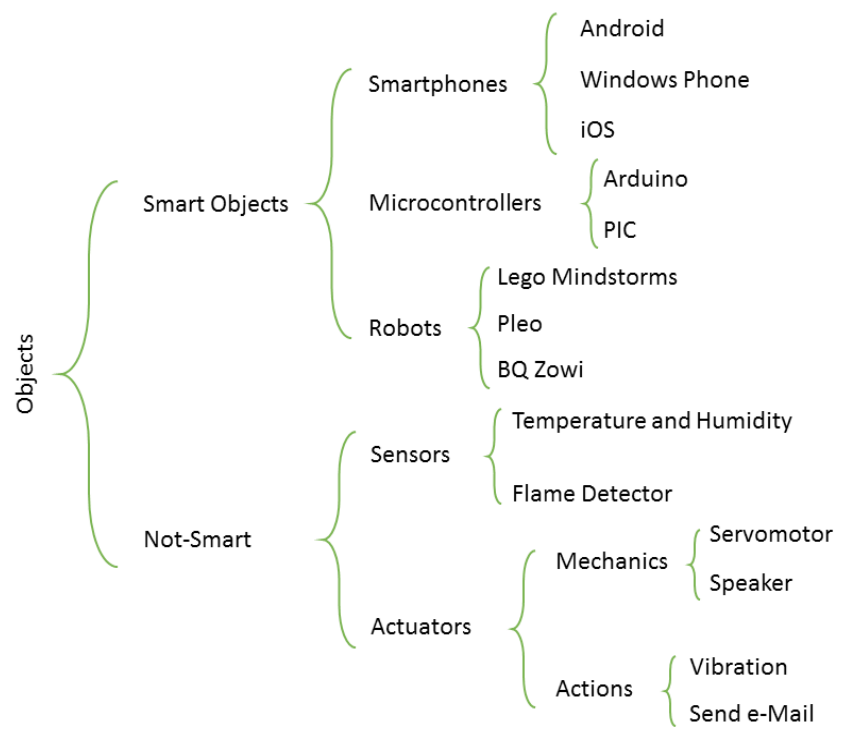

Fig. 1 Composition of objects using examples

\section{SMART OBJeCtS}

The definition of Smart Object depends on its author. Nevertheless, some authors agree with other authors and therefore, we can get a premise of their definitions. Below is our premise which was created using the definitions obtained from [5]-[9].

A Smart Object, also known as Intelligent Product, is a physical element that can be identified throughout its life and interact with the environment and other objects. Moreover, it can act in an intelligent way and independently under certain conditions. Furthermore, Smart Objects have an embedded operating system and they usually can have actuators, sensors, or both [5]. This allows Smart Objects to communicate with other objects, process environment data, and do events. However, there are definitions that differ from the previous which was obtained from [5]-[9].

The definition from [10] is very different from the previous. In [10], they consider as Intelligent Products the objects which are constantly monitoring, which react and adapt to the environment, which have an optimum performance, and which hold an active communication.

In our daily life, we are surrounded by examples of Smart Object and there are also examples in our everyday objects like smartphones, tablets, Smart TVs, microcontrollers like Arduino [5], [11]-[13], and even some coffee pots and some cars are also Smart Objects. Therefore, an object connected to the Internet [14] and capable of manage information [15] can be a Smart Object.

As we can see, Smart Objects can be very different from each other. A smartphone has little in common with a microcontroller and microcomputer. They only have in common some electronic components. Each one has their own sensors and actuators, their own intelligence, and their own operating system when they have one.

Smart Objects can be classified through three dimensions according to [15] and like Fig. 2 shows. This classification is useful to distinguish the different data that a Smart Object can give us about its architecture. Each dimension represents a quality of the intelligence. With the three dimensions, we can determine the intelligence that an object has and the type of Smart Object that it is. The three dimensions are the level of intelligence, the location of the intelligence, and the aggregation level of the intelligence.

\section{A. Level of intelligence}

The first dimension is the level of intelligence. This describes how much intelligent an object can be. It is formed by three levels information handling, notification of the problem, and decision making.

\section{1) Information handling}

The information handling is the capacity of the object to manage the information gathered from sensors, readers, or from any other techniques.

This is the most basic intelligence level and all Smart Object must have it, thus, any Smart Object must be able to manage the information that receives. Otherwise, it would not be a Smart Object and it would be just a Not-Smart Object.

\section{2) Notification of the problem}

The notification of the problem is the ability of an object to notify its owner under certain conditions or when an event occurs like flames detection, an unusual decrease of the temperature, or any other event like these. In this level, the objects do not have free will.

\section{3) Decision making}

The decision making is the highest level of intelligence that an object can have. An object has this level when it has the other two levels and it is able to take decisions by itself. It does not require any type of intervention, thus, it has free will.

\section{B. Location of the intelligence}

The second dimension is the location of the intelligence and is formed by two categories according to [15], but we have added one extra category. Thus, this dimension has three categories: intelligence through the Network, intelligence in the Object, and combined intelligence. Moreover, we added a third level that combines both levels.

\section{1) Intelligence through the Network}

The intelligence through the Network consists in that the intelligence depends totally on an external agent due to the lack of 
intelligence in the object. This agent can be a network where the object is connected, usually known as portal platforms [16], a server that runs the agents or another object that takes decisions or has the global intelligence.

\section{2) Intelligence in the Object}

The intelligence in the Object means that the objects with this level, can process information by themselves, so, they do not need any external agent in order to be intelligent. The platforms that have objects with this level are usually called embedded platforms [16].

\section{3) Combined intelligence}

The combined intelligence is a level that [15] does not include in their classification but they talk about it and they include it in an example graph. In this level, the object has the both intelligences. It has its own intelligence and it is capable of use the intelligence located in the Network. This platforms are usually called surrogated platforms [16].

\section{Aggregation level of the intelligence}

The last dimension is the aggregation level of the intelligence which is formed by three categories. This dimension is useful to describe the objects that are composed of several parts. Depending on the aggregation level we could say that an object is indivisible or every part is independent. For example, we can connect a Raspberry Pi with an Arduino and connect sensors or actuators to both devices. The Not-Smart Objects like the sensors or actuators, do not have their own intelligence but the Raspberry Pi and the Arduino are Smart Objects. Therefore, if we disconnected the Arduino and the Raspberry Pi, they could run independently, whereas if we disconnect the Not-Smart Objects they could not work by themselves.

The two categories are: intelligence in the item, intelligence in the container, and distributed intelligence.

\section{1) Intelligence in the item}

The first category is the intelligence in the item. This category includes the objects that are capable of handling information, notifications and/or decisions. Moreover, if these objects are composed of different components, these components must not be independents. Examples of objects that belong to this category are the smartphones. They are composed of sensors and actuators that cannot be separated because they are embedded.

\section{2) Intelligence in the container}

The second category is the intelligence in the container. The objects of this category must be able to handle information, notifications and/ or decisions and they must know their components in order to work as a proxy between their components and the Internet or the intelligence. Moreover, these objects are capable of working as containers or Smart Objects in spite of removing some of their components. An Arduino with at least two sensors belongs to this category. If we removed a sensor from the Arduino, the Arduino would be able to continue working as container. Another example could be intelligent shelve [15] that notify when a product is out of stock.

\section{3) Distributed Intelligence}

The second category is the distributed intelligence. This category is the fusion between the other two. Here, items and containers have intelligent but, in this case, they can negotiate between themselves according to take the best decision to the object in base on the whole system and the rest of items. This category was added by us because we have worked with object which need this interaction. An example of this category is when you have a Smart Object which is composed by other Smart Objects, for instance, a Raspberry Pi which has connected two Arduinos. In this case, each Arduino has its own intelligence and it can take their own decisions, but sometimes, it has to ask to the Raspberry Pi about some data or the state of the another Arduino to do some action.

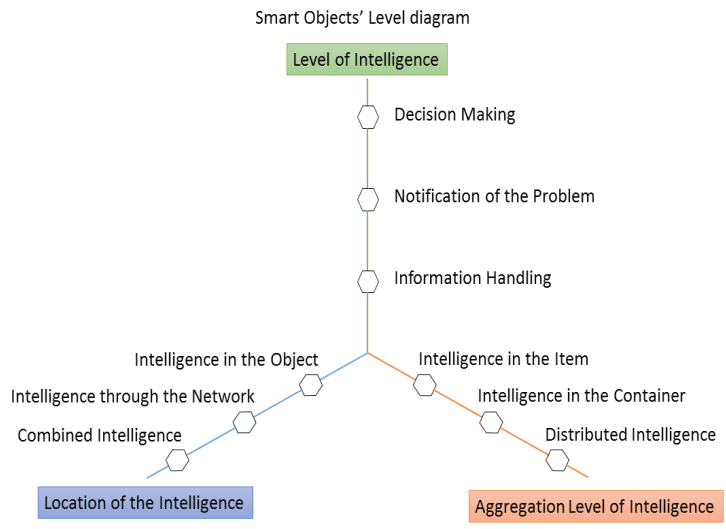

Fig. 2 Classification of the intelligence based on Meyer's classification

From our point of view, the most important type of Smart Objects is the combined intelligence or the intelligence through the network from the dimension location of the intelligence. This type of objects alongside with the Internet of Things, allow adding intelligence to the network and actions according to the data that these objects collect, and services that they offer. In this way, the objects that are connected to the network could have intelligence, or even, be more intelligent.

\section{Application Areas}

Smart Objects are presents in our daily life for a long time. We usually consider that Smart Objects and the Internet of Things go together, although, there are many examples about the usage of Smart Objects without the usage of the IoT.

We can find Smart Objects in different systems on the commercial field in order to control the manufacturing like happens in [6]. Furthermore, in [7], [15], they use Smart Objects in order to improve the distribution and the products management in supply chains to have the products located during all their life cycle.

In the first paper, the authors describe when use different elements like readers in order to know the states of the products, monitoring them, and access to their history. Whereas, in the second paper, they mention an example which we already talked about. They mention intelligent shelves which notify when a product is out of stock.

These kind of applications are very useful to companies because they obtain advantages to improve and avoid problems related with the lack of stock during the all chain of the product life.

Following with possible uses of Smart Objects, another usage is proposed in [17]. This proposal consists in analysing the usage of rented items in order to collect the appropriate quantity of money, and also, punishing improper usage of the object. The system is good for clients as well as for companies. The clients pay exactly for the usage of the product and companies can detect improper usages and be compensated.

Smart Objects can also be used to improve the safety at work. In [17], the authors proposed a system to alert nearby employees about the incorrect and insecure storage of chemical material. The system proposed can be very useful because it allow managing the storage of hazardous substances and avoiding many problems or disasters. 
The medical field is another field where Smart Objects can be used. A research in this field is [18]. In this research, the authors proposed a system that monitor patients with problems. Thanks to systems like this, many human lives could be saved. An example could be the connection of a cardiac pacemaker with a monitoring centre in order to detect, immediately, heart attacks or failures in the pacemaker.

\section{Conclusions}

In this paper, we analysed the differences between Smart objects and Not-Smart Objects. In the literature, we cannot find the exactly differences or we can see as some authors use the both words indistinctly. In fact, this creates a problem to understand the exactly devices that they use.

Smart Objects can be used for resolving a lot of problems. We have showed a few examples of it, from supply chain to security and health.

Notwithstanding, as we say before, objects need a central system to create the interconnection between themselves. According to this goal, we can use a specific system or some Internet of Thing (IoT) platform. We can see some examples in [19] and a classification of the different IoT network types in [20]. The last one contains examples of different IoT platforms which support heterogeneous and ubiquitous objects and interconnect the objects between themselves.

We can see that the combination between Smart Objects and the Internet of Things can offer many advantages and improve the peoples' life because it can interconnect and communicate the different object to create more complex applications. Besides, we have added two new categories to the Meyer's classification in order to adapt to the new type of objects and applications in the Internet of Things.

\section{ACKNOWLEDGMENT}

This work was performed by the 'Ingeniería Dirigida por Modelos MDERG' research group at the University of Oviedo under Contract No. FC-15-GRUPIN14-084 of the research project 'Ingeniería Dirigida Por Modelos MDERG'. Project financed by PR Proyecto Plan Regional.

\section{REFERENCES}

[1] WordReference.com LLC, "WordReference," 2016. [Online]. Available: http://www.wordreference.com/. [Accessed: 17-Feb-2016].

[2] Oxford University Press, "Oxford Dictionaries," 2016. [Online]. Available: http://www.oxforddictionaries.com/. [Accessed: 18-Feb-2016].

[3] Cambridge University Press, "Cambridge Dictionaries Online," 2016. [Online]. Available: http://dictionary.cambridge.org/. [Accessed: 18-Feb2016].

[4] Alphabet Inc., “Google,” 2016. [Online]. Available: http://www.google.es. [Accessed: 17-Feb-2016].

[5] K. A. Hribernik, Z. Ghrairi, C. Hans, and K. Thoben, "Co-creating the Internet of Things - First Experiences in the Participatory Design of Intelligent Products with Arduino," in Concurrent Enterprising (ICE), 2011 17th International Conference on, 2011, pp. 1-9.

[6] D. McFarlane, S. Sarma, J. L. Chirn, C. . Wong, and K. Ashton, "Auto ID systems and intelligent manufacturing control," Eng. Appl. Artif. Intell., vol. 16, no. 4, pp. 365-376, Jun. 2003.

[7] C. Y. Wong, D. McFarlane, A. Ahmad Zaharudin, and V. Agarwal, "The intelligent product driven supply chain," in IEEE International Conference on Systems, Man and Cybernetics, 2002, vol. vol.4, p. 6.

[8] R. Van Kranenburg, D. Caprio, E. Anzelmo, A. Bassi, S. Dodson, and M. Ratto, "The Internet of Things," in 1st Berlin Symposium on Internet and Society, 2011, no. October 2015, p. 84.

[9] M. Kärkkäinen, J. Holmström, K. Främling, and K. Artto, "Intelligent products - a step towards a more effective project delivery chain," Comput. Ind., vol. 50, pp. 141-151, 2003.
[10] O. Ventä, Intelligent products and systems: Technology theme-final report, no. 635. VTT Technical Research Centre of Finland, 2007.

[11] V. Georgitzikis, O. Akribopoulos, and I. Chatzigiannakis, "Controlling Physical Objects via the Internet using the Arduino Platform over 802.15.4 Networks," in Latin America Transactions, IEEE (Revista IEEE America Latina), 2012, vol. 10, no. 3, pp. 1686-1689.

[12] A. Piras, D. Carboni, and A. Pintus, "A Platform to Collect, Manage and Share Heterogeneous Sensor Data," in Networked Sensing Systems (INSS), 2012, pp. 1-2.

[13] Arduino, "Arduino," 2016. [Online]. Available: https://www.arduino.cc/. [Accessed: 09-Feb-2016].

[14] G. M. Lee and J. Y. Kim, "Ubiquitous networking application: Energy saving using smart objects in a home," in 2012 International Conference on ICT Convergence (ICTC), 2012, pp. 299-300.

[15] G. G. Meyer, K. Främling, and J. Holmström, "Intelligent Products: A survey," Comput. Ind., vol. 60, no. 3, pp. 137-148, Apr. 2009.

[16] F. Ramparany and O. Boissier, "Smart devices embedding multi-agent technologies for a pro-active world," in Proc. Uniquitous Computing Workshop, 2002.

[17] G. Kortuem, F. Kawsar, D. Fitton, and V. Sundramoorthy, "Smart objects as building blocks for the Internet of things," IEEE Internet Comput., vol. 14, no. 1, pp. 44-51, Jan. 2010.

[18] I. F. Akyildiz, Weilian Su, Y. Sankarasubramaniam, and E. Cayirci, "A survey on sensor networks," IEEE Commun. Mag., vol. 40, no. 8, pp. 102-114, Aug. 2002.

[19] C. G. García, C. P. García-Bustelo, J. P. Espada, and G. CuevaFernandez, "Midgar: Generation of heterogeneous objects interconnecting applications. A Domain Specific Language proposal for Internet of Things scenarios," Comput. Networks, vol. 64, no. C, pp. 143-158, Feb. 2014.

[20] C. G. García, J. P. Espada, E. R. N. Valdez, and V. G. Diaz, "Midgar: Domain-Specific Language to Generate Smart Objects for an Internet of Things Platform," in 2014 Eighth International Conference on Innovative Mobile and Internet Services in Ubiquitous Computing, 2014, pp. 352-357.

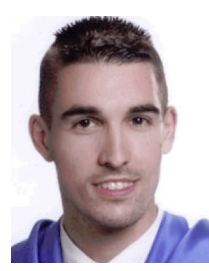

Cristian González García is a Technical Engineering in Computer Systems and M.S. in Web Engineering from School of Computer Engineering of Oviedo in 2011 and 2013 (University of Oviedo, Spain). Currently, he is a Ph.D. candidate in Computers Science. His research interests are in the field of the Internet of Things, Web Engineering, Mobile Devices, and Modelling Software with DSL and MDE.

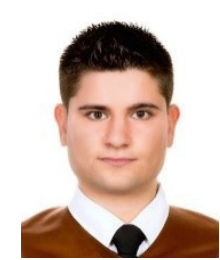

Daniel Meana-Llorián is a Graduated Engineering in Computer Systems from School of Computer engineering of Oviedo in 2014 (University of Oviedo, Spain). Currently, he is a student of M.S. in Web Engineering. His research interests include Mobile technologies, Web Engineering, the Internet of Things, and exploration of emerging technologies related with the previous ones.

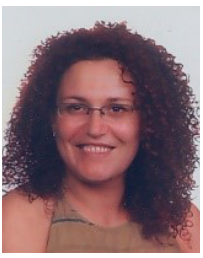

B. Cristina Pelayo G-Bustelo is a Lecturer in the Computer Science Department of the University of Oviedo. Ph.D. from the University of Oviedo in Computer Engineering. Her research interests include Object-Oriented technology, Web Engineering, eGovernment, and Modelling Software with BPM, DSL, and MDA.

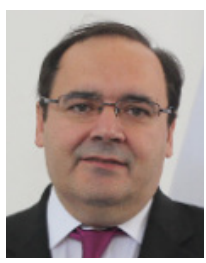

Juan Manuel Cueva Lovelle is a Mining Engineer from Oviedo Mining Engineers Technical School in 1983 (Oviedo University, Spain). Ph. D. from Madrid Polytechnic University, Spain (1990). From 1985 he is Professor at the Languages and Computers Systems Area in Oviedo University (Spain). ACM and IEEE voting member. His research interests include Object-Oriented technology, Language Processors, Human-Computer Interface, Web Engineering, and Modelling Software with BPM, DSL, and MDA. 